Perspective

J Prev Med Public Health 2018;51:326-328 • https://doi.org/10.3961/jpmph.18.150

pISSN 1975-8375 elSSN 2233-4521
Journal of

Preventive Medicine

\& Public Health

\title{
Introduction of Phylodynamics for Controlling the HIV/AIDS Epidemic in Korea
}

\author{
Jong-Myon Bae \\ Department of Preventive Medicine, Jeju National University School of Medicine, Jeju, Korea
}

As over 1000 new cases of HIV/AIDS occur in Korea annually, preventive health programs against HIV/AIDS are urgently needed. Since phylodynamic studies have been suggested as a way to understand how infectious diseases are transmitted and evolve, phylodynamic inferences can be a useful tool for HIV/AIDS research. In particular, phylodynamic models are helpful for dating the origins of an epidemic and estimating its basic reproduction number. Thus, the introduction of phylodynamics would be a highly valuable step towards controlling the HIV/AIDS epidemic in Korea.

Key words: Infectious disease transmission, Molecular epidemiology, Biological evolution, Human immunodeficiency virus, Acquired immunodeficiency syndrome, Epidemics

While the number has been shrinking globally [1], South Korea (hereafter Korea) has seen an increased number of new cases of acquired immunodeficiency syndrome (AIDS) caused by human immunodeficiency virus (HIV) infection since 2013, with more than 1000 new cases occurring annually [2]. This rate of HIV infections poses a major threat to the country's public health, and the actual magnitude of potential healthcare risks may be even greater, as that estimate is based on anonymous and spontaneous surveys [3]. Given these circumstances, it is extremely urgent to reassess the effectiveness of the HIV/AIDS control program put in place by the Korea Centers for Disease Control and Prevention.

While no infections through blood products or blood transfusions have been recorded since 2006, almost all HIV/AIDS in-

Received: July 2, 2018 Accepted: October 22, 2018

Corresponding author: Jong-Myon Bae, MD, PhD

Department of Preventive Medicine, Jeju National University School of Medicine, 102 Jejudaehak-ro, Jeju 63243, Korea

E-mail: jmbae@jejunu.ac.kr

This is an Open Access article distributed under the terms of the Creative Commons Attribution Non-Commercial License (http://creativecommons.org/licenses/by$\mathrm{nc} / 4.0 /$ ) which permits unrestricted non-commercial use, distribution, and reproduction in any medium, provided the original work is properly cited. fections have been spread by sexual contact [2]. Thus, HIV/AIDS prevention in Korea should focus on managing sexual transmission. Epidemiological surveys of new HIV/AIDS patients show that most of them are males but self-identify as heterosexuals, raising questions about the credibility of the survey [3]. But, the government does not share any details about new patients, even for research purposes, underscoring the need to introduce innovative approaches to HIV/AIDS management.

The typical approach taken with new reported cases is to create a social transmission network (STN) based on an epidemiological interview, followed by identifying potential hidden cases by confirming the initial source through a phylogenetic analysis using dynamic molecular analytical techniques [4]. However, it may be difficult to create an STN if any links are missing, or RNA viruses such as HIV could mutate quickly. For the issues, Grenfell et al. [5] proposed the phylodynamic analysis. This method has enabled the calculation of many different contagion indices such as the basic reproductive number, as well as facilitating spatio-temporal studies of transmission routes.

Phylodynamic analysis has become the most important approach used to understand the epidemiology of HIV [6]. Den- 
nis et al. [4] summarized the advantages of phylodynamic studies of HIV infection, as follows [7,8]: First, phylodynamic analysis helps identify the origin of the epidemic by enabling an identification of the transmission route (e.g., injection vs. sexual transmission) in mass outbreaks of HIV. Second, phylodynamic analysis is helpful for understanding the diverse ways of HIV spreads by region, age, and risk behavior, thereby allowing contagion indices to be measured. Third, phylodynamic analysis enables assessments of the effectiveness of management strategies by analyzing how the pace of spread of the disease is reduced by anti-HIV treatment.

In short, phylodynamic analysis facilitates a deeper understanding of the epidemiology of mass outbreaks of infectious disease in many ways, by considering the transmission route within the infected group and mutation levels in individual cases $[9,10]$. Thus, the Korean authorities should adopt the phylodynamic approach urgently to increase the effectiveness of HIV/AIDS prevention. To be more specific, the following 5 suggestions would be taken:

First, an anonymized ID number should be assigned to a new HIV/AIDS patient for phylodynamic analysis, and the study results would be shared among experts.

Second, legal/epidemiological measures should be established to identify potential hidden cases actively where phylodynamic analysis suggests that they exist. This will help improve patients' prognosis by increasing the chance of early treatment and could prevent potential transmission to others caused by ignorance of being infected.

Third, the existing epidemiological survey that simply asks patients whether they engage in homosexual or heterosexual sex should be redesigned to distinguish between different forms of sexual risk behaviors - anal sex (penetrative or nonpenetrative), genital sex (penetrative or non-penetrative), and oral sex (penetrative or non-penetrative) - in order to understand transmission routes more accurately. Since patients may not accurately disclose patterns of sensitive or stigmatized behavior, it is possible to infer sexual risk behaviors based on the results of a phylodynamic analysis.

Fourth, phylodynamic studies should be conducted of existing registered patients, taking into account their known risk behaviors, to identify the unique characteristics of HIV transmission in Korea.

Fifth, phylodynamic studies should be performed in cases of drug resistance to assess current patient management and to seek complementary measures.

\section{CONFLICT OF INTEREST}

The author has no conflicts of interest associated with the material presented in this paper.

\section{SUPPLEMENTAL MATERIALS}

Supplementary Material: Korean version is available at https:// www.jpmph.org/.

\section{ORCID}

Jong-Myon Bae https://orcid.org/0000-0003-3080-7852

\section{REFERENCES}

1. World Health Organization. Global report: UNAIDS report on the global AIDS epidemic 2012; 2012 [cited 2018 Oct 22].

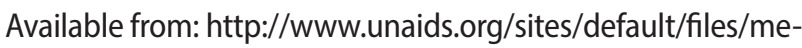
dia_asset/20121120_UNAIDS_Global_Report_2012_with_ annexes_en_1.pdf.

2. Korea Centers for Disease Control and Prevention. 2016 Annual report on the notified HIV/AIDS in Korea. Cheongju: Korea Centers for Disease Control and Prevention; 2017, p. 6-9 (Korean).

3. Bae JM. An interpretation of discrimination prohibition for men who sex with men for preventing HIV/AIDS infection in aspect of public health. Korean Public Health Res 2016;42(2): 69-74 (Korean).

4. Dennis AM, Herbeck JT, Brown AL, Kellam P, de Oliveira T, Pillay $\mathrm{D}$, et al. Phylogenetic studies of transmission dynamics in generalized HIV epidemics: an essential tool where the burden is greatest? J Acquir Immune Defic Syndr 2014;67(2):181-195.

5. Grenfell BT, Pybus OG, Gog JR, Wood JL, Daly JM, Mumford JA, et al. Unifying the epidemiological and evolutionary dynamics of pathogens. Science 2004;303(5656):327-332.

6. Grabowski MK, Redd AD. Molecular tools for studying HIV transmission in sexual networks. Curr Opin HIV AIDS 2014; 9(2):126-133.

7. Volz EM, Koelle K, Bedford T. Viral phylodynamics. PLoS Comput Biol 2013;9(3):e1002947.

8. Volz EM, Frost SD. Inferring the source of transmission with phylogenetic data. PLoS Comput Biol 2013;9(12):e1003397.

9. Colijn C, Gardy J. Phylogenetic tree shapes resolve disease transmission patterns. Evol Med Public Health 2014;2014(1): 96-108. 
10. Norström MM, Karlsson AC, Salemi M. Towards a new paradigm linking virus molecular evolution and pathogenesis: ex- perimental design and phylodynamic inference. New Microbiol 2012;35(2):101-111. 